\title{
Test of protected silver coating on aluminum samples of ARIEL main telescope mirror substrate material
}

Paolo Chioetto, Paola Zupella, Vania Da Deppo, Simone Nordera, Emanuele Pace, et al.

Paolo Chioetto, Paola Zupella, Vania Da Deppo, Simone Nordera, Emanuele Pace, Andrea Tozzi, Gianluca Morgante, Luca Terenzi, Daniele Brienza, Nadia Missaglia, Giovanni Bianucci, Sebastiano Spinelli, Elisa Guerriero, Massimiliano Rossi, Gabriele Grisoni, Colin Bondet, Grégory Chauveau, Caroline Porta, Catherine Grezes-Besset, Giuseppe Malaguti, Giuseppina Micela, "Test of protected silver coating on aluminum samples of ARIEL main telescope mirror substrate material," Proc. SPIE 11852, International Conference on Space Optics - ICSO 2020, 118524L (11 June 2021); doi: $10.1117 / 12.2599794$

SPIE Event: International Conference on Space Optics - ICSO 2021, 2021, Online Only 


\section{International Conference on Space Optics-ICSO 2020}

Virtual Conference

30 March-2 April 2021

Edited by Bruno Cugny, Zoran Sodnik, and Nikos Karafolas
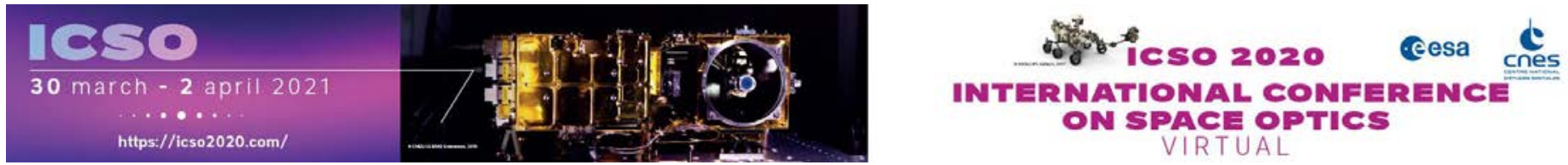

\section{Test of protected silver coating on aluminum samples of ARIEL main telescope mirror substrate material}

\section{Cesa isoporecestings denes}




\title{
Test of protected silver coating on aluminum samples of ARIEL main telescope mirror substrate material
}

\author{
Paolo Chioetto $^{* \text { †a,b,c }}$, Paola Zuppella ${ }^{\dagger a, c}$, Vania Da Deppo ${ }^{\mathrm{a}, \mathrm{c}}$, Simone Nordera ${ }^{\mathrm{a}}$, Emanuele Pace ${ }^{\mathrm{d}}$, \\ Andrea Tozzi ${ }^{\mathrm{e}}$, Gianluca Morgante ${ }^{\mathrm{f}}$, Luca Terenzi ${ }^{\mathrm{f}}$, Daniele Brienza ${ }^{\mathrm{g}}$, Nadia Missaglia ${ }^{\mathrm{h}}$, Giovanni \\ Bianucci $^{\text {h }}$, Sebastiano Spinelli ${ }^{\mathrm{h}}$, Elisa Guerriero ${ }^{\mathrm{j}, \mathrm{k}, \mathrm{h}}$, Massimiliano Rossi ${ }^{\mathrm{h}}$, Gabriele Grisoni ${ }^{\mathrm{h}}$, Colin \\ Bondet $^{\mathrm{i}}$, Gregory Chauveau, Caroline Porta ${ }^{\mathrm{i}}$, Catherine Grezes-Besset ${ }^{\mathrm{i}}$, Giuseppe Malaguti ${ }^{\mathrm{f}}$, \\ Giuseppina Micela ${ }^{j}$
}

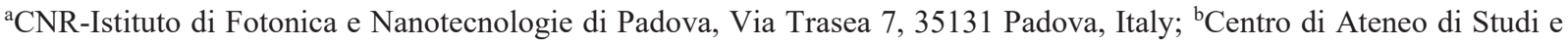
Attività Spaziali “Giuseppe Colombo"- CISAS, Via Venezia 15, 35131 Padova, Italy; 'INAF-Osservatorio Astronomico di Padova, Vicolo dell'Osservatorio 5, 35122 Padova, Italy; ${ }^{\text {d} D i p a r t i m e n t o ~ d i ~ F i s i c a ~ e d ~ A s t r o n o m i a-U n i v e r s i t a ̀ ~ d e g l i ~ S t u d i ~}$

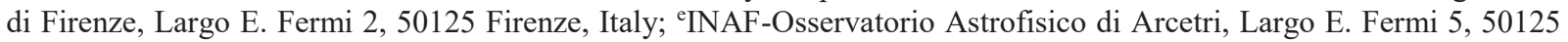
Firenze, Italy; fINAF-Osservatorio di Astrofisica e Scienza dello spazio di Bologna, Via Piero Gobetti 93/3, 40129 Bologna, Italy; gINAF-Istituto di Astrofisica e Planetologia Spaziali, Via Fosso del Cavaliere 100, 00133 Roma, Italy; ${ }^{\mathrm{h}}$ Media Lario S.r.l., Località Pascolo, 23842 Bosisio Parini (Lecco), Italy; ${ }^{\mathrm{i} C I L A S-A r i a n e G r o u p, ~ E t a b l i s s e m e n t ~ d e ~}$ Marseille, 600 avenue de la Roche Fourcade, Pôle ALPHA Sud - Z.I. Saint Mitre, 13400 Aubagne, France; ${ }^{j}$ INAFOsservatorio Astronomico di Palermo, Piazza del Parlamento 1, 90134 Palermo, Italy; ${ }^{2}$ Dipartimento di Fisica e ChimicaUniversità degli Studi di Palermo, Via Archirafi 36, 90128 Palermo, Italy
\end{abstract}

\begin{abstract}
Ariel (Atmospheric Remote-Sensing Infrared Exoplanet Large Survey) has been adopted as the M4 mission for ESA "Cosmic Vision" program. Launch is scheduled for 2029.

ARIEL will study exoplanet atmospheres through transit spectroscopy with a $1 \mathrm{~m}$ class telescope optimized in the waveband between 1.95 and $7.8 \mu \mathrm{m}$ and operating in cryogenic conditions in the temperature range 40-50 K.

Aluminum alloy 6061, in the T651 temper, was chosen as baseline material for telescope mirror substrates and supporting structures, following a trade-off study. To improve mirrors reflectivity within the operating waveband and to protect the aluminum surface from oxidation, a protected silver coating with space heritage was selected and underwent a qualification campaign during Phase B1 of the mission, with the goal of demonstrating a sufficient level of technology maturity.

The qualification campaign consisted of two phases: a first set of durability and environmental tests conducted on a first batch of coated aluminum samples, followed by a set of verification tests performed on a second batch of samples coated alongside a full-size demonstrator of Ariel telescope primary mirror.

This study presents the results of the verification tests, consisting of environmental (humidity and temperature cycling) tests and chemical/mechanical (abrasion, adhesion, cleaning) tests performed on the samples, and abrasion tests performed on the demonstrator, by means of visual inspections and reflectivity measurements.
\end{abstract}

Keywords: 1 -m class space telescope, infrared optics, aluminum mirrors, protected silver coating, coating environmental tests, reflectivity measurements

\footnotetext{
*paolo.chioetto@pd.ifn.cnr.it

$\dagger$ The two authors contributed equally to the article.
} 


\section{INTRODUCTION}

Ariel has been recently adopted as ESA Cosmic Vision Program M4 mission. In its 4-year nominal mission, Ariel will conduct a survey of known exoplanets to characterize their atmospheres through transit spectroscopy in the wavelength band between $0.5 \mu \mathrm{m}$ and $8 \mu \mathrm{m}$.

The Ariel telescope is based on an off-axis, unobscured Cassegrain design with an elliptical primary mirror with an aperture of $1100 \mathrm{~mm}$ (major axis) and $768 \mathrm{~mm}$ (minor axis) and a light collecting area of approximately 0.6 square meters. Telescope performance is diffraction limited at the wavelength of $3 \mu \mathrm{m}$ on a 30" Field of View. The required average telescope throughput is $96 \% \%^{1,2}$. Telescope and instruments will operate at a temperature below $50 \mathrm{~K}$.

Following the heritage of the JWST MIRI instrument ${ }^{3}$, aluminum alloy 6061-T651 has been chosen for mirrors substrates and supporting structures of the telescope, after a trade-off study ${ }^{4}$ on manufacturability and cost.

To protect the mirrors and to improve their reflectivity in the visible section of the operating waveband, a protected silver coating with space heritage from CILAS $\$$ was chosen as baseline.

Although several examples of cryogenic silver-coated aluminum mirrors are found in literature ${ }^{5-7}$, the large size of the primary mirror and its curvature raised concerns on the uniformity of the deposition process and stability of the coating.

An initial study was therefore devised to test optical performance and durability on A16061-T651 substrates, consisting of a qualification campaign on coated aluminum samples ${ }^{8}$, and a verification test on additional samples and on a full-scale demonstrator of Ariel primary mirror denoted PTM.

This paper describes the verification tests, in particular the evaluation of coating performance and durability by means of adhesion tests, reflectivity measurements and environmental tests.

\section{MATERIALS AND PROCESSES}

\subsection{Items Under Test}

The coating verification tests were performed on the following items coated together in the same run:

1. the PTM (Figure 1), a spherical mirror with a radius of curvature of $2401 \mathrm{~mm}$ and an elliptical optical aperture of 1100 x $730 \mathrm{~mm}$, cut from a rolled plate of Al 6061-T651, the current baseline for Ariel telescope mirrors and supporting structure;

2. 6 aluminum samples, shaped as disks $6 \mathrm{~mm}$ thick $25 \mathrm{~mm}$ in diameter, obtained from the same plate from which the PTM substrate had been cut (Figure 2 shows one of the samples being held for visual inspection before the coating run);

3. 2 sets of glass samples measuring $25 \mathrm{~mm}$ and $48 \mathrm{~mm}$ in diameter respectively to serve as reference for spectral reflectivity and for the profilometry measurements.

$¥$ CILAS-ArianeGroup, Etablissement de Marseille, 600 avenue de la Roche Fourcade, Pôle ALPHA Sud - Z.I. Saint Mitre, 13400 Aubagne, France 


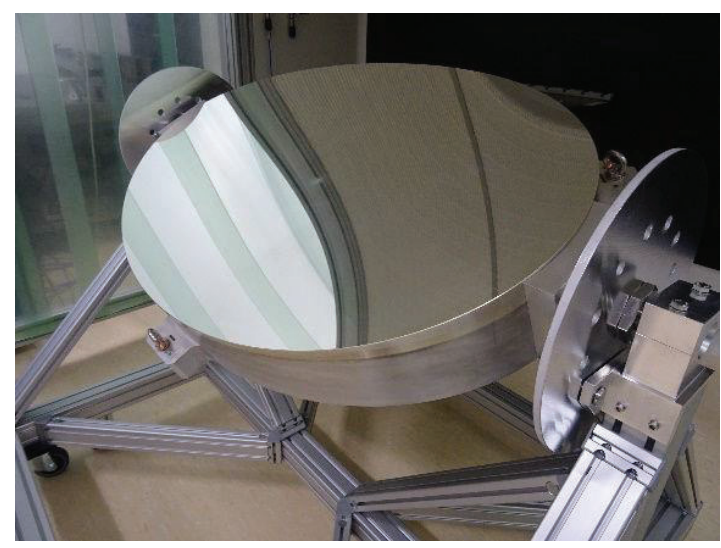

Figure 1. Picture of the PTM mirror mounted on its transport and handling trolley before coating (courtesy of CILAS).

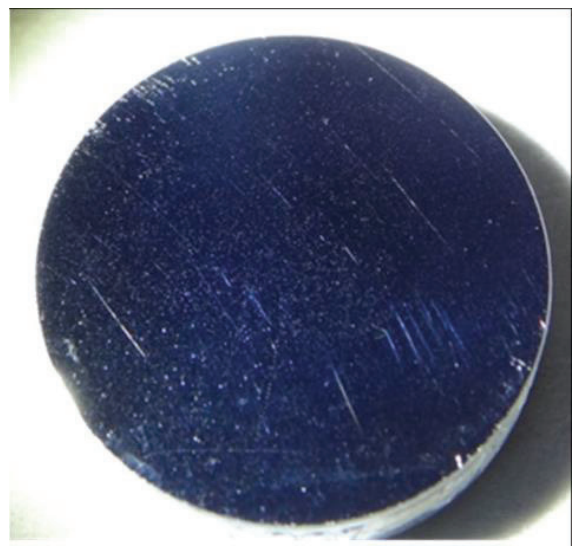

Figure 2. Picture of an aluminum sample used for the qualification during a visual inspection before being coated (courtesy of CILAS).

The roughness of the optical surface on the aluminum samples was measured with a Taylor Hobson CCI White Light Interferometer with magnifications $10 x$ and 50x. All samples measured roughness was within the $10 \mathrm{~nm}$ RMS specification.

The surface roughness of the PTM, measured at 10 different locations on its optical surface, was instead in the range 21.5$26.9 \mathrm{~nm}$ RMS.

The PTM and aluminum samples had been manufactured by MediaLario ${ }^{\S}$

\subsection{Coating Process}

The coating process used by CILAS for this batch is based on physical vapor deposition. The items to be coated are laid on a tray that slides inside a large magnetron sputtering chamber, and moved back and forth beneath a set of cathodes, to minimize deposition inhomogeneities ${ }^{9}$. The process is suited to optical substrates up to $2 \mathrm{~m}$ by $2 \mathrm{~m}$ of footprint and $0.4 \mathrm{~m}$ of thickness ${ }^{10}$.

$\S$ Media Lario S.r.1., Via al Pascolo, 23842 Bosisio Parini (LC), Italy 
The protected silver coating employed for the study consists of three layers: a NiCr adhesion layer of less than $10 \mathrm{~nm}$ of thickness, the silver layer, and a dielectric capping and protection layer. The process had already been qualified on aluminum samples and produces a coating layer measuring on average $350 \mathrm{~nm}$ of thickness, with $10 \%$ uniformity.

The layout of the items on the coating tray is shown in Figure 3.

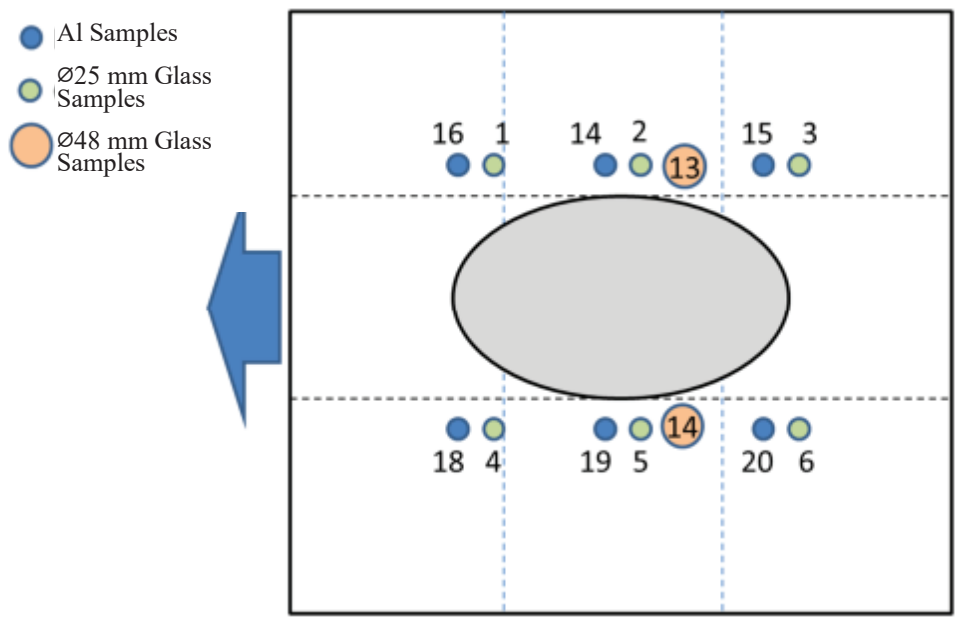

Figure 3. Layout of the items to be coated on the tray of the coating chamber. The blue arrow indicates the direction of insertion into the chamber.

\section{VERIFICATION METHODS}

The tests described in this paper have the purpose of verifying that the performance and durability of the protected silver coating deposited on the PTM are consistent with the results obtained during the qualification phase ${ }^{8}$.

Four of the witness samples deposited together with the PTM were therefore subjected to a series of humidity, temperature, cleaning, abrasion and adhesion tests, evaluated by visual inspection and assessment of the variation of relative spectral reflectivity measurements taken before and after the set of tests.

Additionally, the coating thickness was measured on one of the glass samples, and the durability of the coating on the PTM was assessed through adhesion testing.

The following paragraphs describe the details of the verification methods employed.

\subsection{Visual Inspection}

Visual inspection was performed at CILAS on the optical area of each sample according to ISO standard 9211-4:2012 Inspection of the PTM was performed both by CILAS and MediaLario.

\subsection{Relative Reflectivity Measurements}

Relative reflectivity measurements were performed at CILAS with a Perkin-Elmer Lambda 950 spectrophotometer with a reflectometry accessory in the waveband $500 \mathrm{~nm}-2500 \mathrm{~nm}$ and with an accuracy of $\pm 0.6 \%$ from $500 \mathrm{~nm}$ to $890 \mathrm{~nm}$ and $\pm 1 \%$ above $890 \mathrm{~nm}$.

\subsection{Humidity Test}

The test was realized at CILAS according to ISO 9211-3:2008 ${ }^{12}$ Test \#5 (damp heat). The samples were exposed to a 90\% humidity environment at $55^{\circ} \mathrm{C}\left(+/-3^{\circ} \mathrm{C}\right)$ for 24 hours with a maximum temperature slope of $2^{\circ} \mathrm{C} /$ minute. No condensation was observed on the coated surfaces during the test. 


\subsection{Temperature Cycling Test}

The samples were subjected to 30 cycles between $-40^{\circ} \mathrm{C}$ and $70^{\circ} \mathrm{C}$ with a maximum temperature slope of $2^{\circ} \mathrm{C} / \mathrm{minute}$ and a dwell time of 15 minutes. The test was performed at CILAS.

\subsection{Cleaning Test}

The test was performed at CILAS applying their standard cleaning procedure based on ethanol and acetone solutions, using an optical wipe. The test was repeated 5 times.

\subsection{Abrasion Test}

Test realized at CILAS on one sample, according to ISO 9211-4:2012 ${ }^{11}$, Test Method 01, Severity Level 01.

\subsection{Adhesion Test}

Adhesion tests were performed following ISO Standard 9211-4, Method 02, Severity $02^{11}$.

Tests on samples were performed at CILAS with a cellophane tape, while the test on the optical surface of the PTM was performed at MediaLario using Kapton ${ }^{\circledR}$ tape strips.

\section{TEST RESULTS}

\subsection{Environmental and Mechanical Tests}

One aluminum sample was subjected to the whole sequence of chemical/mechanical tests (cleanability, abrasion and adhesion), while three other samples underwent the environmental set of tests (humidity and temperature cycling) and later the adhesion test. The two remaining aluminum samples have been kept as references for aging.

Results were satisfactory and in line with the outcomes from the coating qualification phase: no discernible signs of coating degradation nor delamination were apparent.

Reflectivity measurements did not highlight any change in performance either, as described in paragraph 4.2.

Adhesion tests on the coated PTM were performed on two separate areas of the mirror, and were also successful with no sign of coating degradation nor visually detectable traces of the coating on the tape strip (Figure 4).

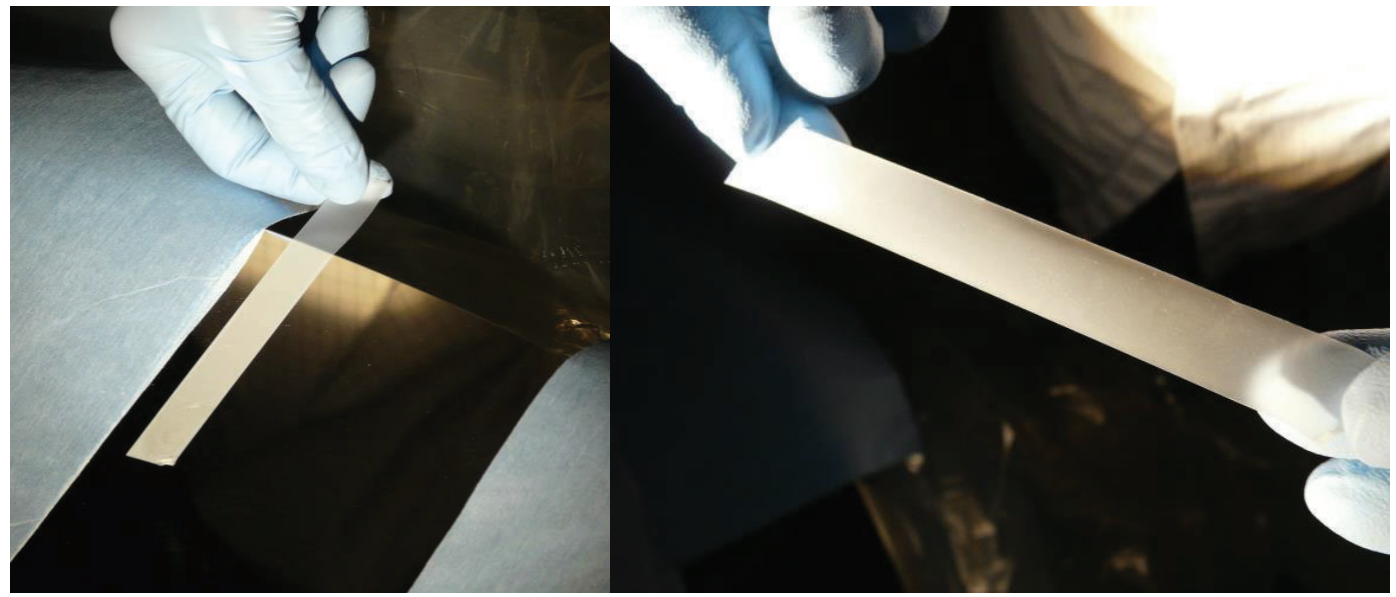

Figure 4. Adhesion test on the PTM mirror. On the left, the tape positioned perpendicular to the edge of the mirror right before lift-off. On the right, the sticky side of the tape after lift-off, showing no detached coating particles (pictures courtesy of MediaLario). 


\subsection{Reflectivity Measurements}

Figure 5 illustrates reflectivity measurements of the samples that underwent mechanical $(\# 18,19,20)$ and cleaning (\#16) tests. Variations between measurements of the same sample are within the accuracy of the measurement instrument.

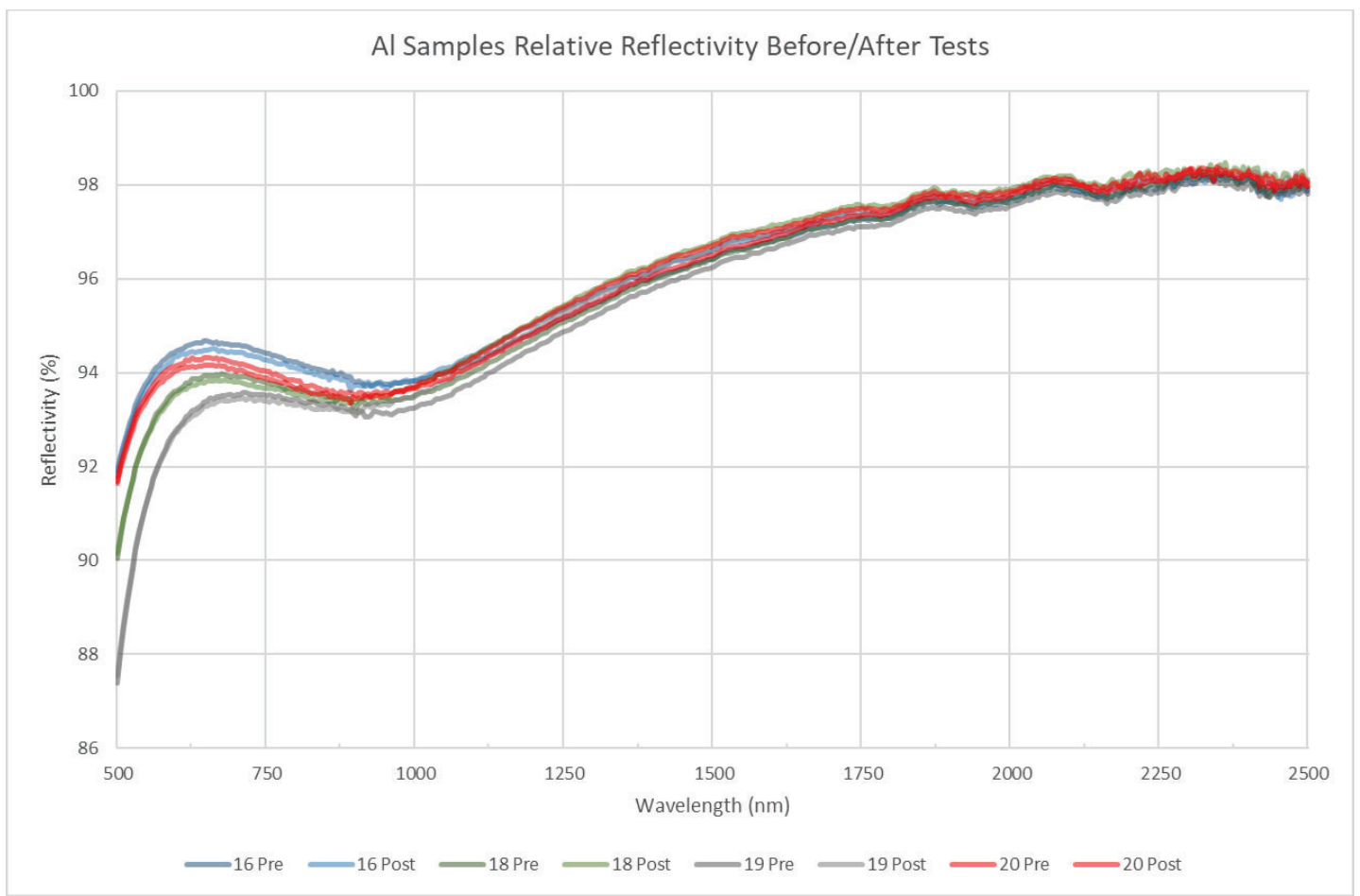

Figure 5. Reflectivity measurements of samples 16, 18, 19 and 20 before ("Pre" in the legend) and after ("Post" in the legend) the tests, showing no significant change.

\subsection{Profilometry}

Glass sample number 14 was partially covered with a mask during coating, and the depth of the resulting ridge was measured with a profilometer in two points. The resulting coating thicknesses were $342 \mathrm{~nm}$ and $345 \mathrm{~nm}$, compatible with measurements taken during the qualification coating run at the same position in the coating chamber of $348 \mathrm{~nm}$.

\section{CONCLUSIONS}

Following the successful qualification of the selected protected silver coating for the aluminum mirrors of the Ariel telescope, a coating test run was performed on the full size demonstrator of the telescope primary mirror (PTM), together with 6 aluminum samples, to further confirm performance and durability of the coating.

Results of adhesion, abrasion, cleaning, humidity and thermal cycling tests performed on the samples and verified by visual inspections and reflectivity measurements showed no alteration in appearance imputable to deterioration or delamination of the coating, nor a degradation in optical performance in the waveband 500-2500 nm.

Adhesion tests performed on the optical surface of the PTM further confirmed the durability of the coating.

These results were considered satisfactory and led to a successful termination of the coating qualification campaign.

Further measurements on the samples will be repeated periodically to assess possible aging deterioration. 


\section{ACKNOWLEDGEMENTS}

This activity has been realized under the Italian Space Agency (ASI) contract with the National Institute for Astrophysics (INAF) n. 2018-22-HH.0, and is partly funded under the ESA contract with Centre Spatial de Liège, Belgium (CSL) and INAF n. 4000126124/18/NL/BW.

\section{REFERENCES}

[1] Da Deppo, V., Middleton, K., Focardi, M., Morgante, G., Claudi, R., Pace, E., Grella, S., Ficai Veltroni, I. and Micela, G., "The afocal telescope optical design and tolerance analysis for the ESA ARIEL mission," Proc. SPIE 10590, International Optical Design Conference 2017, 105901P, SPIE, Denver, United States (2017).

[2] Puig, L., Pilbratt, G., Heske, A., Escudero, I., Crouzet, P.-E., de Vogeleer, B., Symonds, K., Kohley, R., Drossart, P., Eccleston, P., Hartogh, P., Leconte, J., Micela, G., Ollivier, M., Tinetti, G., Turrini, D., Vandenbussche, B. and Wolkenberg, P., "The Phase A study of the ESA M4 mission candidate ARIEL," Exp Astron 46(1), 211-239 (2018).

[3] Kroes, G., Oudenhuysen, A., Meijers, M. and Pel, J.-W., "MIRI-JWST spectrometer main optics opto-mechanical design and prototyping," Proc. SPIE 5877, Optomechanics 2005, 58770P, San Diego, California, USA (2005).

[4] Da Deppo, V., Gianluca, M., Emanuele, P., Middleton, K. and Paul, E., "ARIEL Telescope Material Trade-Off," Technical Paper ARIEL-INAF-PL-TN-004, 10 (2017).

[5] Boccas, M., Vucina, T., Araya, C., Vera, E. and Ahhee, C., "Coating the 8-m Gemini telescopes with protected silver," Proc. SPIE 5494, Optical Fabrication, Metrology, and Material Advancements for Telescopes, 239, USA (2004).

[6] Sheikh, D. A., "Improved silver mirror coating for ground and space-based astronomy," Proc. SPIE 9912 , Advances in Optical and Mechanical Technologies for Telescopes and Instrumentation II, 991239, Edinburgh, United Kingdom (2016).

[7] Schürmann, M., Jobst, P. J., Stenzel, O., Wilbrandt, S., Szeghalmi, A., Bingel, A., Munzert, P., Kaiser, N. and Schwinde, S., "High-reflective coatings for ground and space based applications," SPIE 10563, International Conference on Space Optics — ICSO 2014, 105630M, SPIE, Tenerife, Canary Islands, Spain (2017).

[8] Chioetto, P., Zuppella, P., Da Deppo, V., Pace, E., Morgante, G., Terenzi, L., Brienza, D., Missaglia, N., Bianucci, G., Spinelli, S. M., Guerriero, E., Rossi, M., Bondet, C., Chauveau, G., Porta, C., Grezes-Besset, C., Malaguti, G. and Micela, G., "The primary mirror of the Ariel mission: cryotesting of aluminum mirror samples with protected silver coating," Proc. SPIE 11451, Advances in Optical and Mechanical Technologies for Telescopes and Instrumentation IV, 114511A, SPIE, Online Only, United States (2020).

[9] Savin de Larclause, I., Valette, N., Chauveau, G., Grèzes-Besset, C., Costes, V., Gasc, K. and Lemarquis, F., "PACA2m magnetron sputtering silver coating: a solution for very big mirror dimensions," Proc. SPIE 10563, International Conference on Space Optics - ICSO 2014, 1056308, SPIE, Tenerife, Canary Islands, Spain (2018).

[10] Grèzes-Besset, C., Valette, N., Chauveau, G., Castelnau, M., Mathieu, K., Tatat, M. and Lemarquis, F., "High performance silver coating with PACA2M magnetron sputtering," Proc. SPIE 11180, International Conference on Space Optics - ICSO 2018, 1118083, SPIE, Chania, Greece (2019).

[11] International Organization for Standardization., "International Standard 9211-4: Optics and photonics — Optical coatings — Part 4: Specific test methods" (2012).

[12] International Organization for Standardization., "International Standard 9211-3: Optics and photonics — Optical coatings — Part 3: Environmental durability" (2018). 